\title{
Diphenylarsinic acid sorption mechanisms in soils using batch experiments and EXAFS spectroscopy
}

\author{
Meng Zhu ${ }^{1,3,4}$, Yongming Luo $(\bowtie)^{2,3,4}$, Ruyi Yang ${ }^{1,4}$, Shoubiao Zhou ${ }^{1,4}$, Juqin Zhang ${ }^{1}$, Mengyun Zhang ${ }^{1}$, \\ Peter Christie $^{2}$, Elizabeth L. Rylott ${ }^{5}$
}

1 College of Environmental Science and Engineering, Anhui Normal University, Wuhu 241002, China

2 Key Laboratory of Soil Environment and Pollution Remediation, Institute of Soil Science, Chinese Academy of Sciences, Nanjing 210008, China

3 Key Laboratory of Coastal Environmental Processes and Ecological Remediation, Yantai Institute of Coastal Zone Research,

Chinese Academy of Sciences, Yantai 264003, China

4 Anhui Provincial Engineering Laboratory of Water and Soil Pollution Control and Remediation, Anhui Normal University, Wuhu 241002, China 5 Centre for Novel Agricultural Products, Department of Biology, University of York, York YO10 5DD, UK

\section{H I G H L I G H T S}

- DPAA sorption data was found to fit the Freundlich equation.

- $K_{\mathrm{f}}$ was significantly positive correlated with oxalate-extractable $\mathrm{Fe}_{2} \mathrm{O}_{3}$.

- Ligand exchange was the main mechanism for DPAA sorption on soils.

- Bidentate binuclear and monodentate mononuclear DPAA bonds were identified.

\section{A R T I C L E I N F O}

Article history:

Received 29 September 2019

Revised 18 February 2020

Accepted 20 February 2020

Available online 5 April 2020

Keywords:

Diphenylarsinic acid

EXAFS

Fe (hydr)oxide

Soil organic matter

Sorption mechanism

\section{G R A P H I C A B S T R A C T}

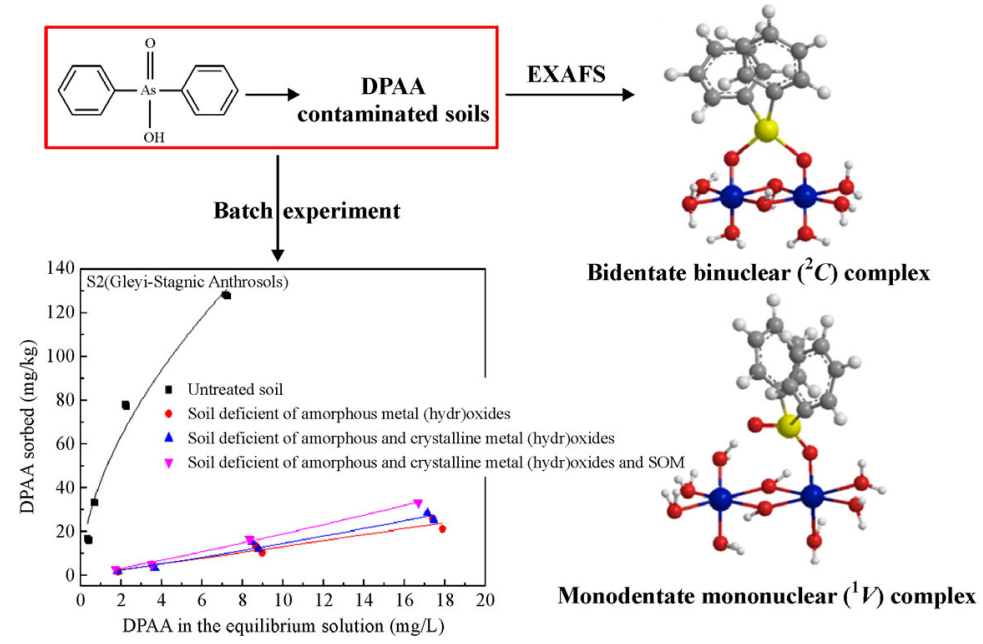

\section{A B S T R A C T}

Diphenylarsinic acid (DPAA) is a phenyl arsenic compound derived from chemical warfare weapons. Macroscopic and microscopic work on DPAA sorption will provide useful information in predicting the partitioning and mobility of DPAA in the soil-water environment. Here, batch experiments and extended X-ray absorption fine structure (EXAFS) spectroscopy were used to investigate the sorption mechanisms of DPAA. The DPAA sorption data from 11 soil types was found to fit the Freundlich equation, and the sorption capacity, $K_{\mathrm{f}}$, was significantly and positively correlated with oxalateextractable $\mathrm{Fe}_{2} \mathrm{O}_{3}$. The $K_{\mathrm{f}}$ values of eight of the 11 untreated soils $(1.51-113.04)$ significantly decreased upon removal of amorphous metal (hydr)oxides (0.51-13.37). When both amorphous and crystalline metal (hydr)oxides were removed from the untreated soils, the $K_{\mathrm{f}}$ values either decreased or slightly increased (0.65-3.09). Subsequent removal of soil organic matter from these amorphous and crystalline metal (hydr)oxide-depleted samples led to further decreases in $K_{\mathrm{f}}$ to $0.02-1.38$, with only one exception (Sulfic Aquic-Orthic Halosols). These findings strongly suggest that ligand exchange reactions with amorphous metal (hydr)oxides contribute most to DPAA sorption on soils. EXAFS data provide further evidence that DPAA primarily formed bidentate binuclear $\left({ }^{2} C\right)$ and monodentate mononuclear $\left({ }^{1} V\right)$ coring-sharing complexes with As-Fe distances of 3.34 and $3.66 \AA$, respectively, on Fe (hydr)oxides. Comparison of these results with earlier studies suggests that ${ }^{2} C$ and ${ }^{1} V$ complexes of DPAA may be favored under low and high surface coverages, respectively, with the formation of ${ }^{1} \mathrm{~V}$ bonds possibly conserving the sorption sites or decreasing the steric hindrance derived from phenyl substituents.

(C) Higher Education Press and Springer-Verlag GmbH Germany, part of Springer Nature 2020 


\section{Introduction}

Diphenylarsinic acid (DPAA) (Fig. S1) is an organic arsenic (As) compound derived from diphenylchloroarsine and diphenylcyanoarsine. These compounds were widely produced and used as chemical warfare agents during World Wars I and II (Kasperek, 1999). After the wars, these agents were naively disposed of via landfill or ocean dumping (Pearson and Magee, 2002; Radke et al., 2014). The leakage of DPAA from chemical warfare agents to soil and groundwater has aroused increasing concern following an incident in Kamisu (Japan), where DPAA-contaminated groundwater led to a clinical syndrome with prominent cerebellar symptoms in many residents (Ishii et al., 2004). The high persistence (Hempel et al., 2009), bioavailability (Arao et al., 2009) and toxicity (Ochi et al., 2004) of DPAA have been amply demonstrated. To fully understand the potential risks of DPAA in the soil-water environment, the sorption mechanisms of DPAA need to be urgently elucidated.

A previous study found that an Fe-rich Acrisol soil had a stronger sorption capacity toward DPAA when compared with a soil organic matter (SOM)-rich Phaeozem (Wang et al., 2013). After the removal of SOM from the Andosol, the amount of DPAA sorbed on the Andosol increased, likely due to the exposure of $\mathrm{Fe} / \mathrm{Al}$ (hydr)oxides that may have been masked by SOM (Maejima et al., 2011). These results suggest that DPAA sorption on soils may be due mainly to ligand exchange reactions with hydroxyl groups on metal (hydr)oxides rather than hydrophobic interactions with SOM. A recent finding is that DPAA exists predominantly as amorphous and crystalline Fe/Al (hydr) oxide associated forms in clay mineral fractions of soils (Zhu et al., 2019a). However, the contributions of Fe/Al (hydr)oxides and their different degrees of crystallinity to DPAA sorption on soils are still unknown. Furthermore, in these studies only a limited number of soil types have been investigated and no direct molecular evidence of DPAA sorption mechanisms is yet available. Therefore, studies involving more diverse soils with varied physicochemical properties and surface characterization techniques are needed to further our understanding of DPAA sorption.

Extended X-ray absorption fine structure (EXAFS) is a powerful in situ technique that has been widely used to identify the coordination environment of inorganic As in soils (Wang and Mulligan, 2008). In recent years there has been research into the use of EXAFS to measure sorbed organic As on $\mathrm{Fe}$ (hydr)oxides. For monomethylarsenate (MMA) and dimethylarsenate (DMA), bidentate binuclear cornering-sharing $\left({ }^{2} C\right)$ complexes were identified on goethite (Shimizu et al., 2011). For DPAA, both ${ }^{2} C$ and monodentate mononuclear cornering-sharing $\left({ }^{1} V\right)$ complexes were observed on ferrihydrite (Tanaka et al., 2014), and similar DPAA ${ }^{2} \mathrm{C}$ bonding was also found on goethite and hematite (Zhu et al., 2019b). However, the molecular environment of DPAA in soils can not be completely represented by the interactions with Fe (hydr)oxides. More EXAFS studies are still needed to elucidate the molecular sorption mechanisms of DPAA in soils.

Here, 11 soil types with a wide range of physicochemical properties were selected for batch sorption experiments. The first objective was to investigate the contributions of amorphous and crystalline metal (hydr) oxides to DPAA sorption on these soils. A highly DPAAcontaminated soil (total As content $>1000 \mathrm{mg} / \mathrm{kg}$ ) collected from north-east China was selected for EXAFS analysis in order to overcome the problem of low signal of organic As compounds and high background noise, and to use material with DPAA concentrations within an optimal range for EXAFS analysis. Consequently, the second objective was to identify the coordination environment of DPAA in a highly DPAA-contaminated soil and provide direct molecular evidence for sorption mechanisms. The present study contributes toward our understanding of the sorption/desorption, bioavailability and transformation of DPAA, and will thus help in the development of appropriate and effective remediation methods for DPAA contamination.

\section{Materials and methods}

\subsection{Reagents}

DPAA (97\% purity) was provided by Wako Pure Chemical Ind. Ltd., Osaka, Japan. Phenylarsonic acid (PAA) (Fig. S1) was purchased from Aladdin Chemical Co. Ltd., Shanghai, China. Standard solutions of MMA and DMA were obtained from the State Center for Standard Materials, Beijing, China. The methanol and formic acid used were of high-performance liquid chromatography (HPLC) grade and other reagents used were of analytical grade. All solutions were prepared with $18.2 \mathrm{M} \Omega / \mathrm{cm}$ ultrapure water (Millipore, Burlington, MA, USA).

\subsection{Soil sampling and analysis}

Eleven types of DPAA-free soils were selected because they are distributed in provinces with reported sites of chemical warfare agents (Deng and Evans, 1997) and because their physicochemical properties range widely. Soils were sampled in each plot from the surface $(0-15 \mathrm{~cm})$ of the soil profile, then air-dried and sieved $(\leqslant 2 \mathrm{~mm})$ in the laboratory. The soil types were classified using the Chinese soils taxonomy described by Gong et al. (2007). The $\mathrm{pH}$, cation exchange capacity (CEC), total P and SOM contents were measured using the standard methods described by $\mathrm{Lu}$ (2000). Four different solutions were used to separately extract $\mathrm{Fe}$ as follows: dithionite-citratebicarbonate (DCB) extraction measured amorphous and 
crystalline $\mathrm{Fe}$ forms (DCB- $\mathrm{Fe}_{2} \mathrm{O}_{3}$ ), ammonium oxalate extraction measured amorphous $\mathrm{Fe}$ forms (oxalate- $\mathrm{Fe}_{2} \mathrm{O}_{3}$ ) (Lu, 2000), sodium pyrophosphate extraction measured Fe-humus complexes (Wada and Higashi, 1976); and HF$\mathrm{HNO}_{3}-\mathrm{HClO}_{4}$ extraction dissolved total $\mathrm{Fe}$ (Pretorius et al., 2006). The concentration of extracted Fe was then determined using phenanthroline colorimetry according to the method described by $\mathrm{Lu}(2000)$. The amorphous and crystalline forms of $\mathrm{Al}\left(\mathrm{DCB}-\mathrm{Al}_{2} \mathrm{O}_{3}\right.$ ) were extracted using $\mathrm{DCB}$, while $\mathrm{HF}-\mathrm{HNO}_{3}-\mathrm{HClO}_{4}$ extraction was used to measure total Al. The concentration of extracted Al was determined by inductively coupled plasma-optical emission spectrometry (ICP-OES, Optima 7000 DV, Perkin Elmer, Waltham, MA, USA). Total As was extracted using $\mathrm{HCl}^{-\mathrm{HNO}_{3}}$ digestion (GB/T 22105.2-2008, Ministry of Environmental Protection of the People's Republic of China) and measured by atomic fluorescence spectrometry (AFS-930, Beijing Jitian Instrument Co., China). Soil particle size distribution was determined using a laser particle size analyzer (Malvern Mastersizer 2000F, Malvern, UK). Recoveries for the determination of SOM (Li et al., 2016), total Fe, $\mathrm{Al}$ (Li et al., 2014) and As were assessed previously in our laboratory through the analysis of geochemical reference materials and ranged from $95 \%$ to $105 \%$. Selected physicochemical properties of the soils are presented in Table 1.

\subsection{Soil preparation}

Samples of the untreated soils $(\leqslant 0.25 \mathrm{~mm})$ were shaken with sodium oxalate-oxalic acid solution $(0.2 \mathrm{~mol} / \mathrm{L}, \mathrm{pH}$ 3.20 , soil:solution $1: 25, \mathrm{w} / \mathrm{v}$ ) in the dark for $2 \mathrm{~h}$ according to the method of $\mathrm{Lu}$ (2000), then washed once with $\mathrm{Na}_{2} \mathrm{CO}_{3}(2 \%$, w/v), three times with $\mathrm{NaCl}(1 \mathrm{~mol} / \mathrm{L})$ and three times with ultra-pure water, respectively, according to the method of Hiradate and Uchida (2004). The treated soil samples were then freeze-dried and sieved to obtain soils deficient of amorphous metal (hydr)oxides. To remove the amorphous and crystalline metal (hydr)oxides, sodium citrate $(0.3 \mathrm{~mol} / \mathrm{L}, \quad 100 \mathrm{~mL})$, sodium bicarbonate $(1.0 \mathrm{~mol} / \mathrm{L}, 12.5 \mathrm{~mL})$ and sodium dithionite $(1 \mathrm{~g})$ were added to 2-g aliquots of the collected soil samples; the mixture was kept at $80^{\circ} \mathrm{C}$ for 15 min with continuous agitation and then centrifuged. The above DCB procedure was followed according to the method of $\mathrm{Lu}(2000)$ and was repeated twice. The treated soil samples were also washed, freeze-dried and sieved to obtain soils deficient of amorphous and crystalline metal (hydr)oxides. Finally, to remove the SOM from the soils deficient of amorphous and crystalline metal (hydr)oxides, these soils were placed in tall beakers and then treated with $\mathrm{H}_{2} \mathrm{O}_{2}(6 \%$, w/v) repeatedly (Hiradate and Uchida, 2004). The treated soil samples were then washed, freeze-dried and sieved to obtain soils deficient of amorphous and crystalline metal (hydr)oxides and SOM.

\subsection{Batch sorption experiments}

DPAA solutions at different initial concentrations $\left(C_{\mathrm{i}}=2\right.$, $4,10$ and $20 \mathrm{mg} / \mathrm{L})$ were prepared in $\mathrm{NaNO}_{3}(0.01 \mathrm{~mol} / \mathrm{L})$ as the background solutions. Untreated or treated soil samples $(2.0 \mathrm{~g})$ and $20 \mathrm{~mL}$ of the DPAA background solutions were placed in Teflon tubes and shaken at $180 \mathrm{r} /$ $\mathrm{min}$ and $25^{\circ} \mathrm{C}$. The mixture was centrifuged at $3000 \mathrm{r} / \mathrm{min}$ for $10 \mathrm{~min}$ and the supernatants were collected after incubation for $72 \mathrm{~h}$, during which time sorption equilibrium was reached, as indicated from our previous study (Wang et al., 2013). Analysis of the equilibrium concentrations of DPAA $\left(C_{\text {eq }}, \mathrm{mg} / \mathrm{L}\right)$ was conducted by HPLCMS/MS equipped with an electrospray ionization source (Zhu et al., 2016b). The experimental data of DPAA sorption was calculated via the following equation:

$$
Q_{\mathrm{ads}}=\left(C_{\mathrm{i}}-C_{\mathrm{eq}}\right) V / m,
$$

where $Q_{\text {ads }}(\mathrm{mg} / \mathrm{kg})$ is the amount of DPAA sorbed, $V$ is the volume of DPAA solution and $m$ is the mass of the dried soil.

$Q_{\text {ads }}$ was plotted against $C_{\text {eq }}$ and analyzed using the Freundlich equation as shown in Eq. (2).

$$
Q_{\mathrm{ads}}=K_{\mathrm{f}} C_{\mathrm{eq}}^{n},
$$

where $K_{\mathrm{f}}$ (sorption coefficient) and $n$ (nonlinearity factor) are the constants related to sorption capacity and intensity, respectively (Petruzzelli et al., 1985).

\subsection{EXAFS analysis}

A soil highly contaminated with DPAA (total As content $>1000 \mathrm{mg} / \mathrm{kg}$ ) was collected from a burial site of chemical warfare weapons in north-east China. A detailed physicochemical analysis of the soil properties was not possible due to the limited amounts of sample available. The highly DPAA-contaminated soil was air-dried and sieved $(\leqslant 0.15 \mathrm{~mm})$, then X-ray absorption near-edge structure (XANES) and EXAFS spectra were collected at the BL14W1 beamline of the Shanghai Synchrotron Radiation Facility (SSRF). The spectra of DPAA, PAA and inorganic As standards were collected in transmission mode, while the spectra of the highly contaminated soil sample, MMA and DMA solutions were collected in fluorescence mode due to their relatively low As concentrations.

Athena and Artemis interfaces from IFEFFIT software were used to analyze the XANES and EXAFS data. The detailed procedure is described in Zhu et al. (2019b), briefly, the spectra were averaged, backgrounds subtracted and then normalized. The radial distribution function (RDF) was obtained through the Fourier transformed (FT) $k^{3}$-weighted $\chi(k)$ EXAFS spectra in the $k$ range of 2.0 $10.0 \AA^{-1}$. The peaks in RDF were related to shells around the central As atom which were fitted by specific atom 


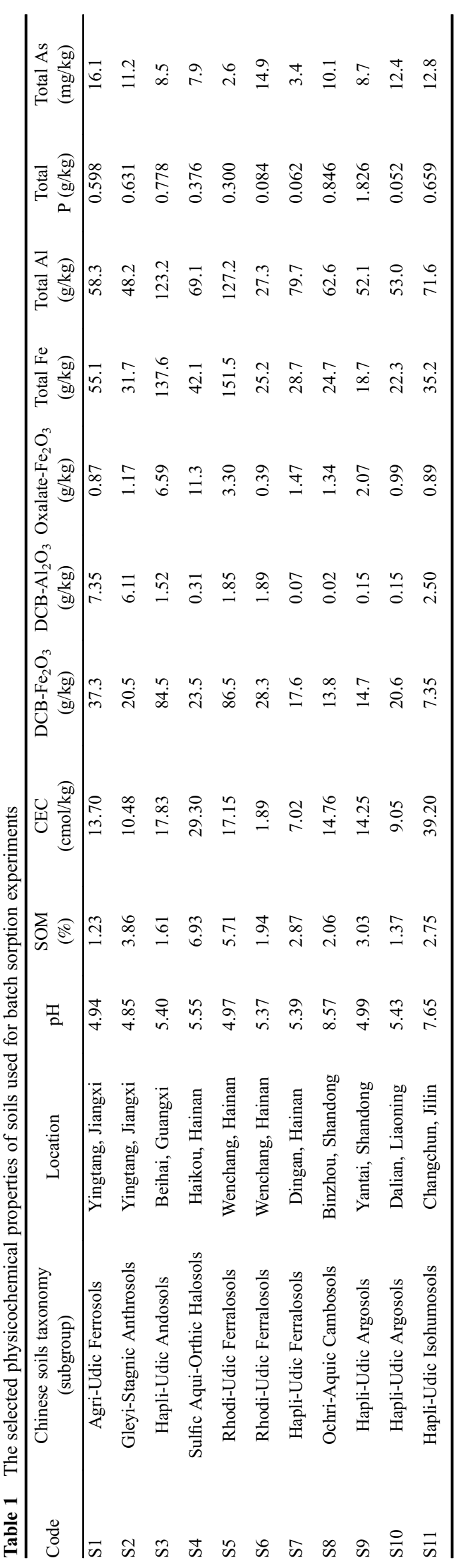


type, coordination number $(\mathrm{CN})$ of atoms in the shell, inter-atomic distance $(R)$, and Debye-Waller factor $\left(\sigma^{2}\right)$. For each EXAFS spectrum the first, second and thirdneighboring contributions to the FT were extracted and back-transformed to obtain partial EXAFS spectra. The CNs for As-O and As-C paths were held constant during the EXAFS fitting, and the $\sigma^{2}$ for different As-C paths was set as one variable for the highly DPAA-contaminated soil sample and another variable for the DPAA standard, in order to reduce the number of adjustable parameters. The quality of the fit was represented by the $R$-factor which is given as the mean square difference between the measured and fit data on a point-by-point basis. An $R$-factor less than 0.02 generally indicates a good fit (Kelly et al., 2008). Athena was also used for linear combination fitting (LCF) of the As K-edge XANES spectra, and the LCF results of the highly DPAA-contaminated soil were used to determine the relative proportion each reference compound contributed to the soil sample.

\subsection{Statistical analysis}

DPAA sorption isotherms were processed using the Origin 8.5 software package (OriginLab Corporation, Northampton, USA). Pearson's correlation was used to determine the relationships between $K_{\mathrm{f}}$ and the selected soil physicochemical properties. All statistical analyses were carried out using the IBM SPSS Statistics 20.0 software
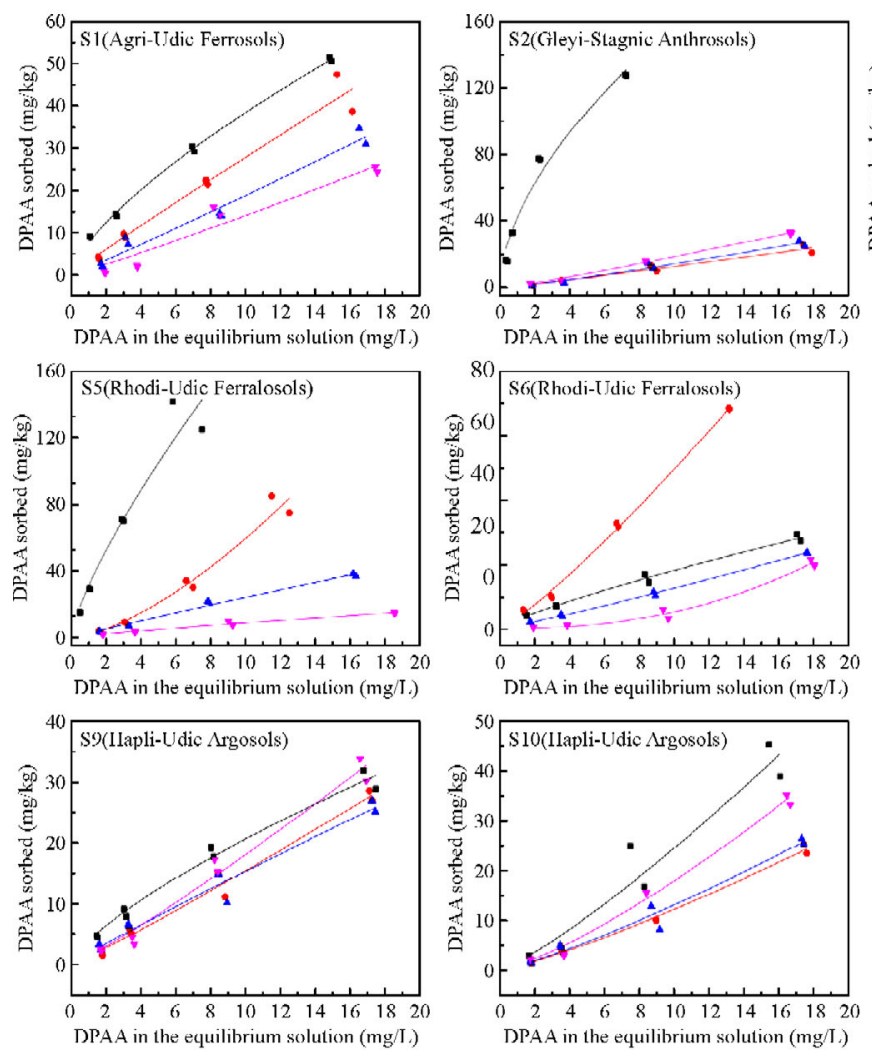

package (SPSS, Chicago, IL, USA).

\section{$3 \quad$ Results and discussion}

\subsection{Correlation between DPAA sorption and soil properties}

The sorption isotherms of DPAA on 11 types of untreated soils are shown in Fig. 1. It was found that the amounts of DPAA sorbed increased with increasing equilibrium concentration of DPAA, and the data obtained from batch experiments were well described by the Freundlich equation $\left(R^{2}>0.93\right)$. All fitting parameters are presented in Table 2. Previous studies also reported that DPAA sorption data on Acrisols, Phaeozems (Wang et al., 2013), Fluvisols and Andosols (Maejima et al., 2011) were best represented by the Freundlich isotherms. The Pearson correlation matrix of $K_{\mathrm{f}}$ with the selected soil physicochemical properties is listed in Table 3. It can be seen that $K_{\mathrm{f}}$ was significantly and positively correlated with oxalate- $\mathrm{Fe}_{2} \mathrm{O}_{3}$ ( $r=0.911, p<0.01)$, indicating that ligand exchange reactions with hydroxyl groups on amorphous Fe (hydr) oxides contribute significantly to DPAA sorption on soils. However, no significant correlation was found between $K_{\mathrm{f}}$ and DCB- $\mathrm{Fe}_{2} \mathrm{O}_{3}(r=0.226, p>0.05)$ or total $\mathrm{Fe}(r=0.257$, $p>0.05$; Table 3 ), reflecting the importance of the speciation of Fe (hydr)oxides in DPAA sorption on soils. Additionally, a less significant correlation was observed
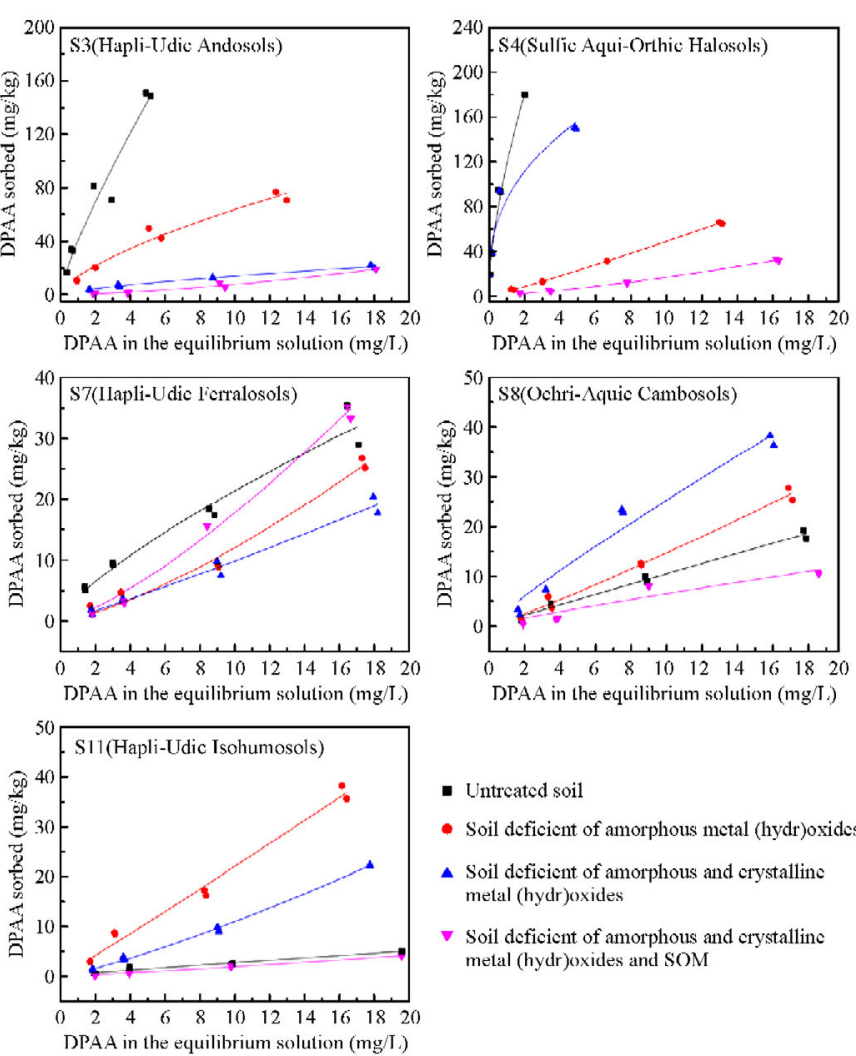

- Untreated soil

- Soil deficient of amorphous metal (hydr)oxides

- Soil deficient of amorphous and erystalline metal (hydr)oxides

v Soil deficient of amorphous and erystalline metal (hydr)oxides and SOM

Fig. 1 Comparison of DPAA sorption isotherms between untreated and treated soils for 11 soil types. 
between $K_{\mathrm{f}}$ and SOM $(r=0.770, p<0.01$; Table 3), suggesting that SOM promotes DPAA sorption either through hydrophobic interactions (Arroyo-Abad et al., 2011), and/or through inhibiting the crystallization of $\mathrm{Fe}$ (hydr)oxides (Schwertmann, 1966; ThomasArrigo et al., 2018) and hence increasing the contributions of amorphous Fe (hydr)oxides. A significant Pearson's correlation between SOM and oxalate- $\mathrm{Fe}_{2} \mathrm{O}_{3}(r=0.643, p<0.05$; Table 3 ) further supports the latter hypothesis but more experimental data are required to give a definitive answer.

\subsection{Role of metal (hydr)oxides in DPAA sorption on soils}

Figure 1 shows that in eight of the 11 untreated soils, the sorption isotherms of DPAA were shifted downward by the removal of amorphous metal (hydr)oxides, an effect not observed for S6, S8 and S11; the Rhodi-Udic Ferralosols, Ochri-Aquic Cambosols and Hapli-Udic Isohumosols, respectively. The $K_{\mathrm{f}}$ values in these eight soils lacking amorphous metal (hydr)oxides (0.505-13.370) were significantly lower than in the untreated soils (1.512113.042), especially in S4 where the $K_{\mathrm{f}}$ value decreased from 113.042 to 3.682 (Table 2). These results agree with the Pearson's correlation analysis in that $K_{\mathrm{f}}$ was strongly and positively correlated with oxalate- $\mathrm{Fe}_{2} \mathrm{O}_{3}$ (Table 3 ), indicate that amorphous metal (hydr)oxides provide the majority of the sorption sites for DPAA on the soils. The findings in the present study correspond well with our previous study, which demonstrated that amorphous ferrihydrite was more efficient in sorption of DPAA compared with crystalline goethite and hematite (Zhu et al., 2019b). This effect may have resulted from the high surface area, and thereby high sorption capacity, of amorphous metal (hydr)oxides compared with crystalline ones (Dixit and Hering, 2003). For soil samples S6, S8 and S11, the $K_{\mathrm{f}}$ values from untreated samples $(2.754,1.046$ and 0.323 , respectively) were only slightly increased by the removal of amorphous metal (hydr)oxides (3.225, 1.070 and 2.006, respectively) (Table 2). This contrasting effect, when compared with the other eight soils might be explained by the low availability of amorphous Fe (hydr) oxides in S6, S8 and S11, and is possibly due to the covering of the low content of oxalate- $\mathrm{Fe}_{2} \mathrm{O}_{3}$ by the relatively high content of SOM (Table 1) (Prietzel et al., 2007; Regelink et al., 2014); once the amorphous metal (hydr)oxides have been removed, the DPAA-sorption sites of SOM and clay minerals become exposed and the DPAA is available for extraction.

The sorption isotherms of DPAA on soils after the removal of both amorphous and crystalline metal (hydr) oxides are presented in Fig. 1, and the Freundlich parameters are listed in Table 2. It can be seen that in S1, S2, S3, S6 and S11 the $K_{\mathrm{f}}$ values in soils deficient of both amorphous and crystalline metal (hydr)oxides decreased by $13 \%-80 \%$ compared with the soils deficient of amorphous-only metal (hydr)oxides (Table 2). These results strongly suggest that both amorphous and crystalline metal (hydr)oxides contribute to DPAA sorption on soils. However, in S5, S7, S8, S9 and S10 the $K_{\mathrm{f}}$ values in soils deficient of both amorphous and crystalline metal (hydr)oxides (0.744-3.092) increased only slightly compared with their soils deficient of amorphous-only metal (hydr)oxides (0.505-1.780) (Table 2). The discrepancy may be explained by differences in the removal efficiency of more active, amorphous metal (hydr)oxides using DCB and oxalate treatments (McKeague and Day, 1966). In addition, in all the soils mentioned above (S1-S3, S5-S11) the $K_{\mathrm{f}}$ values decreased to $\leqslant 3.092$ by the removal of amorphous and crystalline metal (hydr)oxides from untreated soils, suggesting that amorphous and crystalline metal (hydr)oxides provide most sorption sites for DPAA. However, the $K_{\mathrm{f}}$ value of S4 remained at 84.256 despite the

Table 2 Freundlich equation parameters for DPAA sorption on 11 types of soils $\mathrm{s}^{\text {a) }}$

\begin{tabular}{|c|c|c|c|c|c|c|c|c|c|c|c|c|}
\hline \multirow[t]{2}{*}{ Code } & \multicolumn{3}{|c|}{ Untreated soil } & \multicolumn{3}{|c|}{$\begin{array}{l}\text { Soil deficient of amorphous } \\
\text { metal (hydr)oxides }\end{array}$} & \multicolumn{3}{|c|}{$\begin{array}{l}\text { Soil deficient of amorphous and } \\
\text { crystalline metal (hydr)oxides }\end{array}$} & \multicolumn{3}{|c|}{$\begin{array}{l}\text { Soil deficient of amorphous and crystal- } \\
\text { line metal (hydr)oxides and SOM }\end{array}$} \\
\hline & $K_{f}$ & $n$ & $R^{2}$ & $K_{f}$ & $n$ & $R^{2}$ & $K_{f}$ & $n$ & $R^{2}$ & $K_{f}$ & $n$ & $R^{2}$ \\
\hline$\overline{\mathrm{S} 1}$ & 7.432 & 0.712 & 0.997 & 3.020 & 0.962 & 0.959 & 1.619 & 1.062 & 0.965 & 1.114 & 1.096 & 0.923 \\
\hline S2 & 42.292 & 0.567 & 0.970 & 1.132 & 1.054 & 0.955 & 0.984 & 1.161 & 0.969 & 1.377 & 1.130 & 0.995 \\
\hline S3 & 39.737 & 0.800 & 0.950 & 13.370 & 0.675 & 0.966 & 2.679 & 0.708 & 0.986 & 0.205 & 1.561 & 0.981 \\
\hline S4 & 113.042 & 0.634 & 0.984 & 3.682 & 1.102 & 0.999 & 84.256 & 0.373 & 0.913 & 0.742 & 1.330 & 0.997 \\
\hline S5 & 30.694 & 0.762 & 0.936 & 1.780 & 1.523 & 0.962 & 2.649 & 0.956 & 0.983 & 1.214 & 0.857 & 0.975 \\
\hline S6 & 2.754 & 0.816 & 0.986 & 3.225 & 1.184 & 0.997 & 0.989 & 1.104 & 0.998 & 0.024 & 2.334 & 0.981 \\
\hline S7 & 3.701 & 0.758 & 0.954 & 0.505 & 1.372 & 0.976 & 0.744 & 1.117 & 0.974 & 0.819 & 1.331 & 0.988 \\
\hline S8 & 1.046 & 0.988 & 0.984 & 1.070 & 1.124 & 0.987 & 3.092 & 0.902 & 0.961 & 0.798 & 0.897 & 0.877 \\
\hline S9 & 3.636 & 0.736 & 0.982 & 1.249 & 1.088 & 0.978 & 1.745 & 0.940 & 0.960 & 1.324 & 1.131 & 0.974 \\
\hline S10 & 1.512 & 1.207 & 0.931 & 0.724 & 1.223 & 0.989 & 0.777 & 1.223 & 0.967 & 0.890 & 1.301 & 0.988 \\
\hline S11 & 0.323 & 0.916 & 0.972 & 2.006 & 1.039 & 0.982 & 0.649 & 1.226 & 0.998 & 0.123 & 1.175 & 0.979 \\
\hline
\end{tabular}

Notes: a) $K_{\mathrm{f}}$ means sorption coefficient, $n$ means nonlinearity factor, $R^{2}$ means correlation coefficient of Freundlich isotherm. 
removal of amorphous and crystalline metal (hydr)oxides (Table 2) and this might be due to the following reasons: 1) the incomplete removal of amorphous and crystalline metal (hydr)oxides by the DCB treatment; removal can alter depending on variation of aging and crystallization of soil ( $\mathrm{Lu}, 2000)$; 2) the high content of Fe-humus complexes (Fig. S2) in S4, which have a strong affinity toward phenyl As (Peng et al., 2016), and this organiccomplexed $\mathrm{Fe}$ can be extracted by pyrophosphate and oxalate but not DCB (McKeague et al., 1971). The $K_{\mathrm{f}}$ value of S4 decreased to only 0.724 with the removal of amorphous and crystalline metal (hydr)oxides and SOM (Table 2), which further strengthens the latter hypothesis.

Based on these results, it can be inferred that DPAA, which has two As-O groups, would have been sorbed by amorphous and crystalline metal (hydr)oxides to make inner-sphere complexes via ligand exchange reactions. It is widely known that metal (hydr)oxides also provide most sorption sites for As in soils (Cancès et al., 2005), and thus these As compounds compete with DPAA for the same sorption sites. Our results show that $K_{\mathrm{f}}$ was negatively correlated with total As $(r=-0.279)$, although such correlation did not reach a significant level $(p>0.05)$ (Table 3). We propose that the increased steric hindrance caused by phenyl substitution, and thereby the decreased availability of hydroxyl groups on metal (hydr)oxides (Adamescu et al., 2010), may have more influence on DPAA sorption compared with the competitive sorption of As in soils.

\subsection{Role of soil organic matter in DPAA sorption on soils}

Figure 1 shows that the sorption isotherms of DPAA were shifted downward by the removal of SOM from all soils deficient of amorphous and crystalline metal (hydr)oxides, except for those of S2, S7, S9 and S10. Concurrently, the $K_{\mathrm{f}}$ values decreased for most soils deficient of amorphous and crystalline metal (hydr)oxides with the removal of SOM but increased slightly for those of S2, S7 and S10 (Table 2). Many discrepancies in the role of SOM in the sorption of phenyl As have been reported and factors related to the role of SOM, namely competitive sorption (Zhu et al., 2016a), covering sorption sites on Fe/Al (hydr) oxides (Maejima et al., 2011), forming SOM-Fe-As complexes (Fu et al., 2016) and hydrophobic interactions (Arroyo-Abad et al., 2011) should be considered, and the contribution of each factor to the sorption of phenyl As may depend on soil type.

Overall, the changes in the $K_{\mathrm{f}}$ values of untreated and treated soils (Table 2) show that amorphous metal (hydr) oxides contributed more to DPAA sorption compared with crystalline metal (hydr)oxides and SOM. Despite the limited contribution of SOM to DPAA sorption, there was a significant positive correlation between $K_{\mathrm{f}}$ and SOM (Table 3) as pointed out earlier. These results agree with well-established evidence that SOM can inhibit the crystallization of $\mathrm{Fe} / \mathrm{Al}$ (hydr)oxides (ThomasArrigo et al., 2018) and thus indirectly promote DPAA sorption on soils.

3.4 Direct evidence for DPAA association with Fe (hydr) oxides by EXAFS

XANES has been widely used as a powerful tool to identify the speciation of As in contaminated soils (Cancès et al., 2008). The XANES spectra of As-containing reference compounds and a highly DPAA-contaminated soil are presented in Fig. 2(a). The LCF of As XANES spectra (Fig. 2(b)) indicated a mixture of DPAA and inorganic $\mathrm{As}(\mathrm{V})$, with the percentages of $88.7 \%$ and $11.3 \%$, respectively, demonstrating that As was present mainly as DPAA in the highly contaminated soil.

The $k^{3}$-weighted, whole EXAFS functions of the highly DPAA-contaminated soil and the corresponding RDFs are

Table 3 Pearson correlation matrix ( $r=$ Pearson correlation coefficient) of DPAA sorption with selected physicochemical properties of soils $(n=$ $11)^{\text {a) }}$

\begin{tabular}{|c|c|c|c|c|c|c|c|c|c|c|c|}
\hline & $K_{\mathrm{f}}$ & $\mathrm{pH}$ & SOM & CEC & DCB- $\mathrm{Fe}_{2} \mathrm{O}_{3}$ & DCB- $\mathrm{Al}_{2} \mathrm{O}_{3}$ & Oxalate- $\mathrm{Fe}_{2} \mathrm{O}_{3}$ & Total Fe & Total Al & Total As & Total P \\
\hline$K_{\mathrm{f}}$ & 1 & & & & & & & & & & \\
\hline $\mathrm{pH}$ & -0.277 & 1 & & & & & & & & & \\
\hline SOM & $0.770 * *$ & -0.216 & 1 & & & & & & & & \\
\hline CEC & 0.367 & 0.369 & 0.394 & 1 & & & & & & & \\
\hline DCB- $\mathrm{Fe}_{2} \mathrm{O}_{3}$ & 0.226 & -0.122 & 0.138 & -0.056 & 1 & & & & & & \\
\hline DCB- $\mathrm{Al}_{2} \mathrm{O}_{3}$ & -0.021 & -0.550 & -0.158 & -0.036 & 0.097 & 1 & & & & & \\
\hline Oxalate- $\mathrm{Fe}_{2} \mathrm{O}_{3}$ & $0.911 * *$ & 0.030 & $0.643 *$ & 0.436 & 0.326 & -0.285 & 1 & & & & \\
\hline Total Fe & 0.257 & -0.047 & 0.231 & 0.151 & 0.970 ** & 0.088 & 0.361 & 1 & & & \\
\hline Total Al & 0.243 & 0.135 & 0.282 & 0.317 & $0.803^{* *}$ & -0.155 & 0.417 & $0.898 * *$ & 1 & & \\
\hline Total As & -0.279 & 0.006 & -0.577 & -0.044 & -0.378 & 0.505 & -0.373 & -0.451 & $-0.679^{*}$ & 1 & \\
\hline Total P & -0.098 & 0.028 & -0.064 & 0.219 & -0.119 & -0.013 & 0.008 & -0.079 & -0.036 & 0.032 & 1 \\
\hline
\end{tabular}

Notes: a) * means significant level at $p<0.05$, ** means significant level at $p<0.01$. 
included in Figs. 3(a) and 3(b), respectively. The $k^{3}$ weighted EXAFS spectra of the three nearest neighboring shells were extracted, and the results are presented in Figs. 4(a) and 4(b), respectively. The two fitting approaches yielded similar results, which are listed in Table 4. The $k^{3}$-weighted EXAFS spectra of DPAA, together with the contributions of the first and second shells, can be found in our previous study (Zhu et al., 2019a).

When the RDF of the highly DPAA-contaminated soil (Fig. 3(b)) was compared with that of the DPAA standard (Zhu et al., 2019a), both yielded an intense peak at 1-2 $\AA$, which is related to the contributions of As-O and As- $\mathrm{C}_{1}$ (the nearest $\mathrm{C}$ ) in the mixed, first-neighbor shell. The fitted inter-atomic distances for As-O and $\mathrm{As}^{-\mathrm{C}_{1}}$ (Table 4) were 1.69 and $1.89 \AA$, respectively. A broad peak was located at
2-3.3 $\AA$ in the RDF of the soil sample (Fig. 3(b)) in contrast to the DPAA standard, and the second shell was fitted with four $\mathrm{C}$ atoms at $\mathrm{As}-\mathrm{C}_{2}$ (the second-nearest $\mathrm{C}$ ) distance of $2.79 \AA$ (Table 4). The fitted As-O, As- $\mathrm{C}_{1}$ and As- $\mathrm{C}_{2}$ distances in the present study correspond well with the EXAFS data (Tanaka et al., 2014; Zhu et al., 2019a, 2019b) and density functional theory (DFT) calculations (Tanaka et al., 2014) reported in the literature.

However, fitting the highly DPAA-contaminated soil sample only with these two shells yielded a relatively high $R$-factor (0.039) and other abnormal fitting parameters (data not shown), and inclusion of As-Fe paths at distances around 3.4 and $3.6 \AA$ was necessary to obtain a good fit of the RDF with the $R$-factor of 0.017 (Table 4). Consequently, our EXAFS data indicate that the third-shell peak arises from the scattering from neighboring $\mathrm{Fe}$ atoms, and
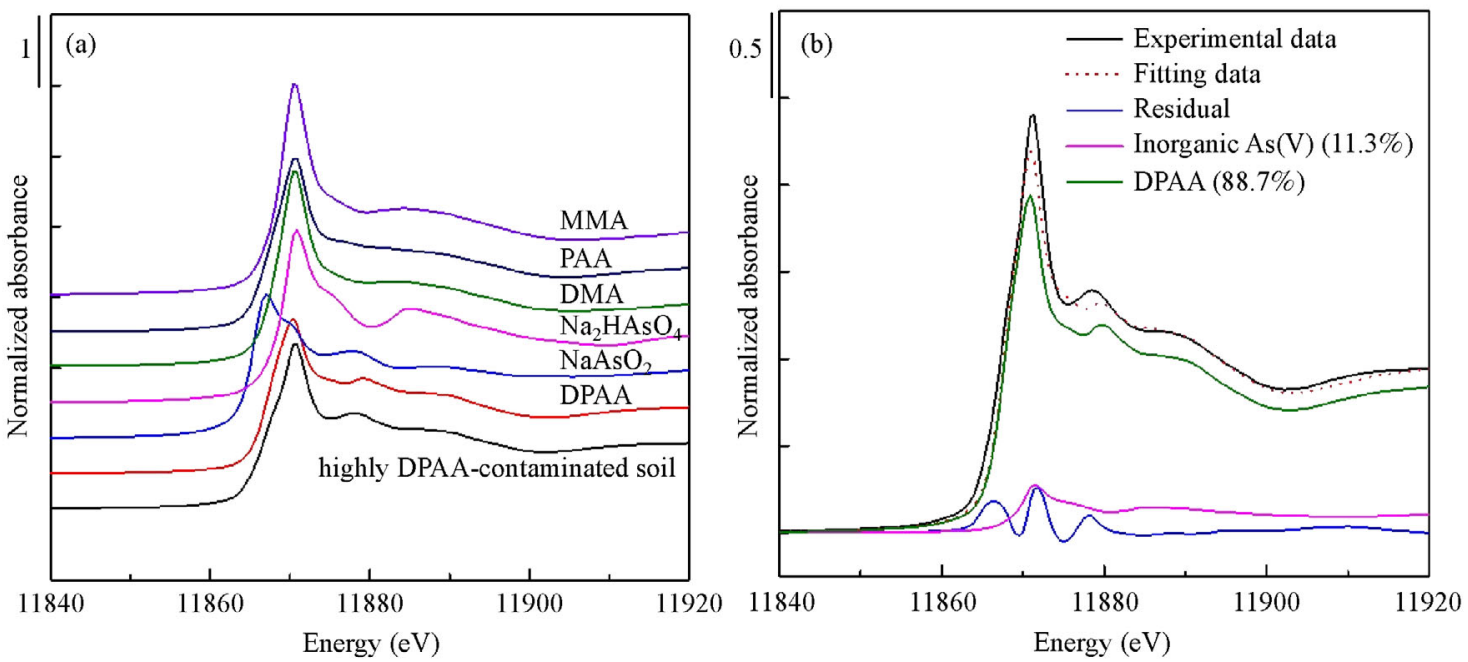

Fig. 2 (a) The normalized As K-edge XANES spectra of the highly DPAA-contaminated soil and As-containing reference compounds, and (b) linear combination fit for the highly DPAA-contaminated soil, spectra denoting the fractional contributions of the components were used to generate the fitted spectra.
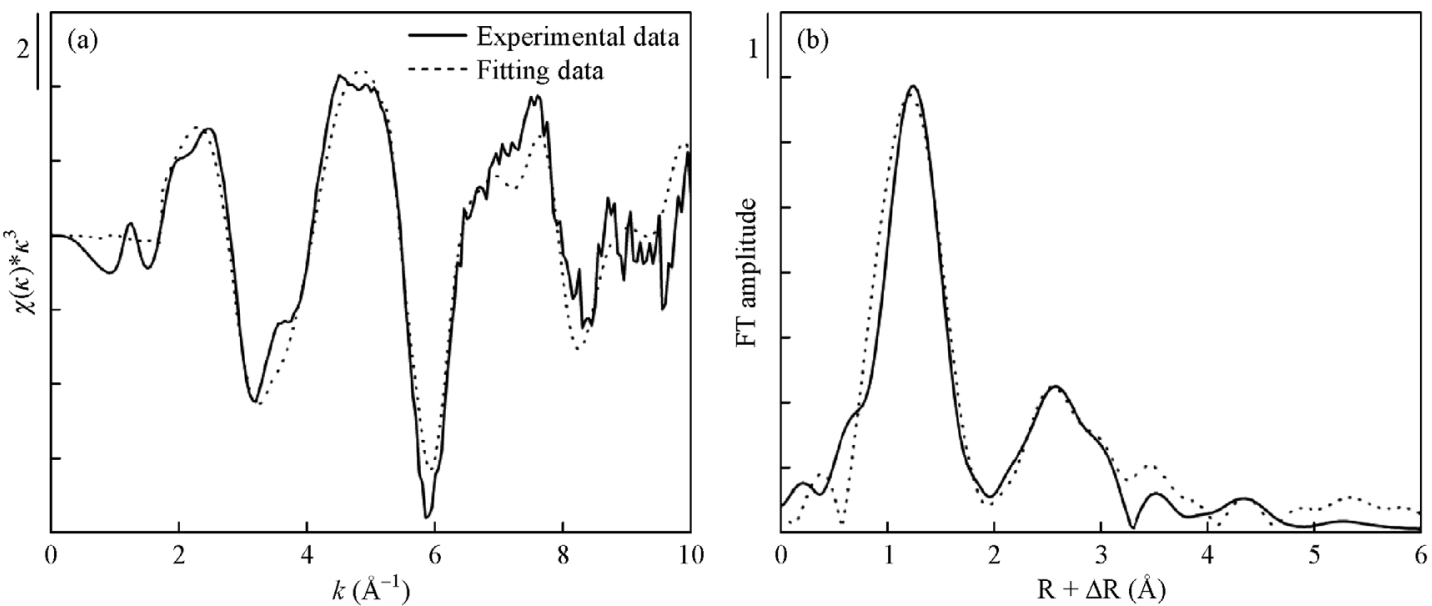

Fig. 3 (a) The As K-edge EXAFS spectra and (b) $k^{3}$-weighted Fourier-transformed (FT) spectra of the highly DPAA-contaminated soil. 

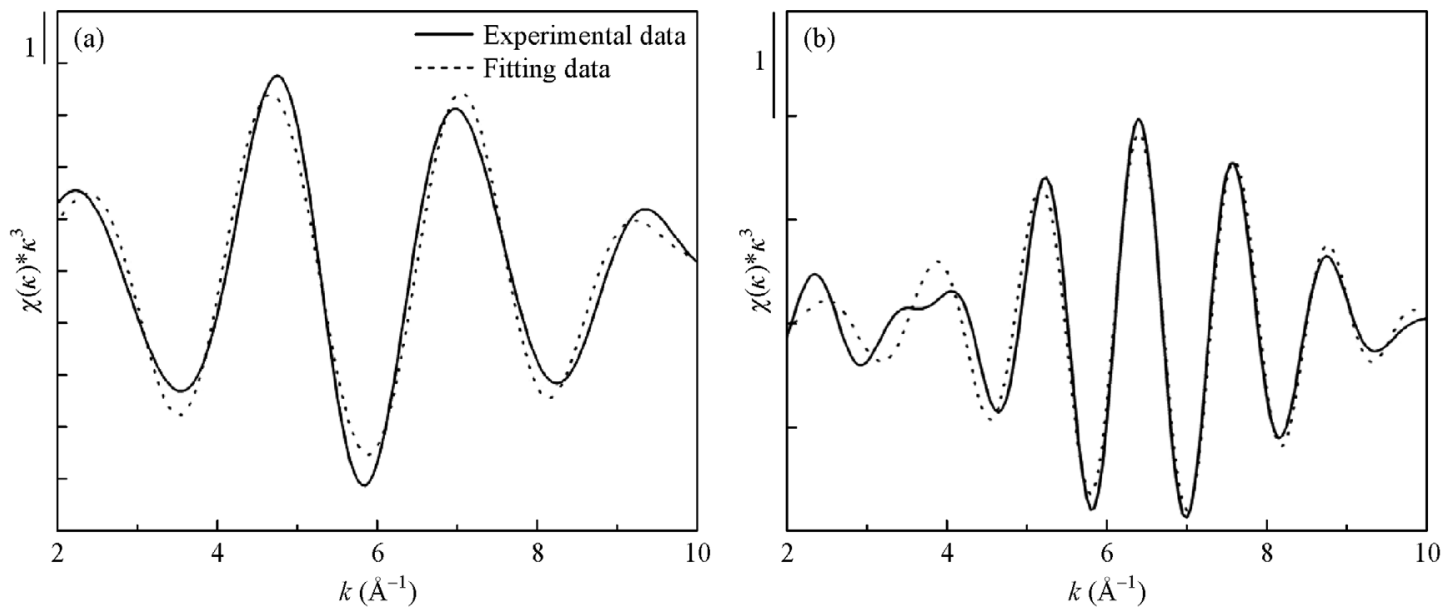

Fig. 4 The partial $k^{3}$-weighted $\chi(k)$ EXAFS functions of (a) first, (b) second and third neighboring shells of As for the highly DPAAcontaminated soil.

Table 4 Structural data on molecular environment of As derived from EXAFS data ${ }^{\text {a) }}$

\begin{tabular}{|c|c|c|c|c|c|c|}
\hline Sample & Shell & $\overline{\mathrm{CN}}$ & Atomic distance $(\AA)$ & $\sigma^{2}\left(\AA^{2}\right)$ & $R$-factor & Reference \\
\hline \multirow[t]{3}{*}{$\overline{\text { DPAA }}$} & As-O & 2.00 & $1.70(0.02)$ & 0.0030 & \multirow[t]{3}{*}{0.009} & \multirow[t]{3}{*}{ Zhu et al. (2019a) } \\
\hline & As- $\mathrm{C}_{1}$ & 2.00 & $1.99(0.03)$ & 0.0010 & & \\
\hline & As- $\mathrm{C}_{2}$ & 4.00 & $2.87(0.06)$ & 0.0010 & & \\
\hline \multirow{5}{*}{$\begin{array}{l}\text { Highly DPAA-contaminated } \\
\text { soil }\end{array}$} & As-O & 2.00 & $1.69(0.03)$ & 0.0040 & \multirow[t]{5}{*}{0.017} & \multirow[t]{5}{*}{ This study } \\
\hline & $\mathrm{As}-\mathrm{C}_{1}$ & 2.00 & $1.89(0.13)$ & 0.0030 & & \\
\hline & $\mathrm{As}-\mathrm{C}_{2}$ & 4.00 & $2.79(0.13)$ & 0.0030 & & \\
\hline & As- $\mathrm{Fe}_{1}$ & $2.15(0.27)$ & $3.34(0.16)$ & 0.0030 & & \\
\hline & As- $-\mathrm{Fe}_{2}$ & $1.43(0.32)$ & $3.66(0.17)$ & 0.0030 & & \\
\hline
\end{tabular}

Notes: a) Numbers in bold font were fixed during fitting and uncertainties are listed in parentheses, CN means coordination number, $\sigma^{2}$ means Debye-Waller factor, $R$ factor indicates quality of the fit.

the highly DPAA-contaminated soil sample was satisfactorily fitted by $2.15 \mathrm{Fe}$ atoms at an $\mathrm{As}-\mathrm{Fe}_{1}$ distance of $3.34 \AA$, and $1.43 \mathrm{Fe}$ atoms at an $\mathrm{As}-\mathrm{Fe}_{2}$ distance of $3.66 \AA$ (Table 4). The fitted As-Fe distances at 3.34 and $3.66 \AA$ correspond to As linked to the surface of Fe (hydr)oxides by forming ${ }^{2} C$ and ${ }^{1} V$ complexes, respectively, according to Fendorf et al. (1997). These types of DPAA complexes have been observed in DPAA sorption onto various $\mathrm{Fe}$ minerals (Tanaka et al., 2014; Zhu et al., 2019b) and clay mineral fractions of soils (Zhu et al., 2019a).

Inclusion of an As-Al contribution at $3.2 \AA$, whether replacing or in combination with As-Fe paths, did not improve the fit of the highly DPAA-contaminated soil sample (data not shown). These results strongly suggest that $\mathrm{Fe}$, rather than $\mathrm{Al}$ (hydr)oxides, contribute more to DPAA sorption on soils. The EXAFS data provide molecular evidence that DPAA sorption on soils can be mainly ascribed to its ligand exchange reactions with $\mathrm{Fe}$ (hydr)oxides, and this result is in agreement with the finding that 1) the $K_{\mathrm{f}}$ values were significantly and positively correlated with oxalate- $\mathrm{Fe}_{2} \mathrm{O}_{3}$ (Table 3) and
2) amorphous and crystalline metal (hydr)oxides provided most of the sorption sites for DPAA on soils.

3.5 Comparison with previous EXAFS data for inorganic and phenyl arsenics

Our EXAFS data demonstrate the formation of DPAA ${ }^{2} C$ and ${ }^{1} V$ complexes in a highly DPAA-contaminated soil. It is well recognized that inorganic As mainly forms ${ }^{2} C$ and ${ }^{1} V$ complexes on $\mathrm{Fe}$ (hydr)oxides, with ${ }^{1} V$ complexes favored at low surface coverage but ${ }^{2} \mathrm{C}$ at high surface coverage (Fendorf et al., 1997; Morin et al., 2008). The DFT calculations further demonstrate that ${ }^{2} C$ complexes were more energetically favored over ${ }^{1} V$ complexes for inorganic As sorption onto Fe (hydr)oxides (Sherman and Randall, 2003). However, for DPAA, when comparing the results between soil clay mineral fractions (Zhu et al., 2019a) and a highly DPAA-contaminated soil, similarly in a comparison between ferrihydrite conducted by our previous study (Zhu et al., 2019b) and that by Tanaka et al. (2014), both ${ }^{2} C$ and ${ }^{1} V$ complexes were observed at a 
relatively high surface coverage, while only ${ }^{2} C$ complexes were seen at a relatively low surface coverage. It is suggested that DPAA ${ }^{2} C$ complexes may be favored at low surface coverage while ${ }^{1} V$ complexes appear to be in a greater proportion at higher surface coverage. The formation of ${ }^{1} V$ complexes at high surface coverage can, on the one hand, conserve the sorption sites on Fe (hydr) oxides, and on the other hand, decrease the steric hindrance derived from two phenyl substituents. A similar steric effect was seen for $p$-arsanilic ( $p$ AsA; Fig. S1), and has been reported elsewhere (Depalma et al., 2008), in which only ${ }^{1} V$ bonds were found on $\mathrm{Fe}$ (hydr)oxides (Adamescu et al., 2014). The steric hindrance derived from two phenyl groups may explain the contrasting effects of surface coverage on the sorption structure of DPAA and inorganic As. More experimental data and computational work are still required to predict the thermodynamic favorability of different DPAA complexes on various surfaces found in the soil-water environment, and to further elucidate the overall sorption mechanisms of DPAA.

\subsection{Environmental relevance of the findings}

The current study reveals that the content of oxalateextractable $\mathrm{Fe}_{2} \mathrm{O}_{3}$ was one of the major factors determining the sorption capacity of DPAA in soils. This result suggests that, on the one hand, an increased activation of Fe (hydr) oxides in the soil-water environment, likely where organic matter accumulates (Schwertmann, 1966), would possibly decrease the mobility and bioavailability of DPAA; whereas, on the other hand, DPAA associated with amorphous $\mathrm{Fe}$ (hydr)oxides, which are more likely to be reduced in organic-rich environment compared with crystalline ones (Weber et al., 2010), could represent the potentially mobilizable pool of DPAA under flooded conditions. These results highlight the need for further exploration on the temporal change of DPAA concentrations in organic- and Fe-rich soils under drying/wetting conditions. In addition, contrasting effects of SOM on DPAA sorption on soils were observed in the present study, and it is suggested that SOM may alter DPAA sorption through competitive sorption, covering sorption sites on $\mathrm{Fe} / \mathrm{Al}$ (hydr)oxides, hydrophobic interactions, forming SOM-Fe-DPAA and inhibition of $\mathrm{Fe} / \mathrm{Al}$ (hydr)oxide crystallization, and this needs to be taken into consideration when predicting the fate, mobility and bioavailability of DPAA in organic-rich soils.

\section{Conclusions}

Here, we have investigated the sorption mechanisms of DPAA on 11 soil types. The results show that the $K_{\mathrm{f}}$ values were significantly decreased by the removal of amorphous metal (hydr)oxides from untreated soils, and thereby indicate that amorphous Fe (hydr)oxides were one of the major factors determining DPAA sorption on soils. EXAFS data provide direct evidence that DPAA mainly formed ${ }^{2} C$ and ${ }^{1} V$ complexes on $\mathrm{Fe}$ (hydr)oxides in a highly DPAA-contaminated soil. A combination of batch experiments and EXAFS results demonstrate the importance of DPAA association with Fe (hydr)oxides in its sorption on soils. It is suggested that DPAA ${ }^{1} V$ complexes appear to be at a greater proportion at high surface coverage, which can on the one hand conserve sorption sites on Fe (hydr)oxides, and on the other hand decrease the steric hindrance derived from phenyl substituents.

Acknowledgements This work was supported by the National Natural Science Foundation of China (Grant Nos. 41807117 and 41230858), the Cultivation Project on Excellent Undergraduates' Thesis (design, create) of Anhui Normal University (No. pyjh2018487) and the Innovation and Entrepreneurship Training Project for College Students (No. 201910370072). The EXAFS data were obtained at the BL14W1 beamline at the Shanghai Synchrotron Radiation Facility (SSRF). We thank Dr. Xu Wang at SSRF for help with EXAFS data analysis.

Electronic Supplementary Material Supplementary material is available in the online version of this article at https://doi.org/10.1007/s11783-020$1237-\mathrm{x}$ and is accessible for authorized users.

\section{References}

Adamescu A, Hamilton I P, Al-Abadleh H A (2014). Density functional theory calculations on the complexation of $p$-arsanilic acid with hydrated iron oxide clusters: Structures, reaction energies, and transition states. Journal of Physical Chemistry A, 118(30): 56675679

Adamescu A, Mitchell W, Hamilton I P, Al-Abadleh H A (2010). Insights into the surface complexation of dimethylarsinic acid on iron (oxyhydr)oxides from ATR-FTIR studies and quantum chemical calculations. Environmental Science \& Technology, 44(20): 78027807

Arao T, Maejima Y, Baba K (2009). Uptake of aromatic arsenicals from soil contaminated with diphenylarsinic acid by rice. Environmental Science \& Technology, 43(4): 1097-1101

Arroyo-Abad U, Elizalde-Gonzállez M P, Hidalgo-Moreno C M, Mattusch J, Wennrich R (2011). Retention of phenylarsenicals in soils derived from volcanic materials. Journal of Hazardous Materials, 186(2-3): 1328-1334

Cancès B, Juillot F, Morin G, Laperche V, Alvarez L, Proux O, Hazemann J H, Brown G E Jr, Calas G (2005). XAS evidence of As (V) association with iron oxyhydroxides in a contaminated soil at a former arsenical pesticide processing plant. Environmental Science \& Technology, 39(24): 9398-9405

Cancès B, Juillot F, Morin G, Laperche V, Polya D, Vaughan D J, Hazemann J L, Proux O, Brown G E Jr, Calas G (2008). Changes in arsenic speciation through a contaminated soil profile: A XAS based study. Science of the Total Environment, 397(1-3): 178-189

Deng H, Evans P O M (1997). Social and environmental aspects of abandoned chemical weapons in China. Nonproliferation Review, 4 (3): 101-108 
Depalma S, Cowen S, Hoang T, Al-Abadleh H A (2008). Adsorption thermodynamics of $p$-arsanilic acid on iron (oxyhydr) oxides: In-situ ATR-FTIR studies. Environmental Science \& Technology, 42(6): 1922-1927

Dixit S, Hering J G (2003). Comparison of arsenic(V) and arsenic(III) sorption onto iron oxide minerals: Implications for arsenic mobility. Environmental Science \& Technology, 37(18): 4182-4189

Fendorf S, Eick M J, Grossl P, Sparks D L (1997). Arsenate and chromate retention mechanisms on goethite. 1. Surface structure. Environmental Science \& Technology, 31(2): 315-320

Fu Q L, He J Z, Blaney L, Zhou D M (2016). Sorption of roxarsone onto soils with different physicochemical properties. Chemosphere, 159: 103-112

Gong Z T, Zhang G L, Chen Z L (2007).Pedogenesis and Soil Taxonomy. Beijing: Science Press (in Chinese)

Hempel M, Daus B, Vogt C, Weiss H (2009). Natural attenuation potential of phenylarsenicals in anoxic groundwaters. Environmental Science \& Technology, 43(18): 6989-6995

Hiradate S, Uchida N (2004). Effects of soil organic matter on $\mathrm{pH}$ dependent phosphate sorption by soils. Soil Science and Plant Nutrition, 50(5): 665-675

Ishii K, Tamaoka A, Otsuka F, Iwasaki N, Shin K, Matsui A, Endo G, Kumagai Y, Ishii T, Shoji S, Ogata T, Ishizaki M, Doi M, Shimojo N (2004). Diphenylarsinic acid poisoning from chemical weapons in Kamisu, Japan. Annals of Neurology, 56(5): 741-745

Kasperek T (1999). Chemical Weapons Dumped in the Baltic Sea. Lysomice: Europejskie Centrum Edukacyjne

Kelly S D, Hesterberg D, Ravel B (2008). Part 5-Mineralogical methods. In: Ulery A L, Drees L R, eds. Methods of Soil Analysis. Madison: American Society of Agronomy Press, 387-463

Li Y, Zhang H B, Chen X B, Tu C, Luo Y M, Christie P (2014). Distribution of heavy metals in soils of the Yellow River Delta: Concentrations in different soil horizons and source identification. Journal of Soils and Sediments, 14(6): 1158-1168

Li Y, Zhang H B, Tu C, Fu C C, Xue Y, Luo Y M (2016). Sources and fate of organic carbon and nitrogen from land to ocean: Identified by coupling stable isotopes with $\mathrm{C} / \mathrm{N}$ ratio. Estuarine, Coastal and Shelf Science, 181: 114-122

Lu R K (2000).Analysis Methods of Soil Agricultural Chemistry. Beijing: China Agricultural Science and Technology Press (in Chinese)

Maejima Y, Murano H, Iwafune T, Arao T, Baba K (2011). Adsorption and mobility of aromatic arsenicals in Japanese agricultural soils. Soil Science and Plant Nutrition, 57(3): 429-435

McKeague J A, Brydon J E, Miles N M (1971). Differentiation of forms of extractable iron and aluminum in soils. Soil Science Society of America Journal, 35(1): 33-38

McKeague J A, Day J (1966). Dithionite- and oxalate-extractable Fe and $\mathrm{Al}$ as aids in differentiating various classes of soils. Canadian Journal of Soil Science, 46(1): 13-22

Morin G, Ona-Nguema G, Wang Y H, Menguy N, Juillot F, Proux O, Guyot F, Calas G, Brown G E Jr (2008). Extended X-ray absorption fine structure analysis of arsenite and arsenate adsorption on maghemite. Environmental Science \& Technology, 42(7): 23612366

Ochi T, Suzuki T, Isono H, Kaise T (2004). In vitro cytotoxic and genotoxic effects of diphenylarsinic acid, a degradation product of chemical warfare agents. Toxicology and Applied Pharmacology, 200(1): 64-72

Pearson G S, Magee R S (2002). Critical evaluation of proven chemical weapon destruction technologies (IUPAC Technical Report). Pure and Applied Chemistry, 74(2): 187-316

Peng Y, Wei W, Zhou H, Ge S, Li S Y, Wang G X, Zhang Y (2016). Iron humate as a novel adsorbent for $p$-arsanilic acid removal from aqueous solution. Journal of Dispersion Science and Technology, 37 (11): 1590-1598

Petruzzelli G, Guidi G, Lubrano L (1985). Ionic strength effect on heavy metal adsorption by soil. Communications in Soil Science and Plant Analysis, 16(9): 971-986

Pretorius W, Weis D, Williams G, Hanano D, Kieffer B, Scoates J (2006). Complete trace elemental characterisation of granitoid (USGS G-2, GSP-2) reference materials by high resolution inductively coupled plasma-mass spectrometry. Geostandards and Geoanalytical Research, 30(1): 39-54

Prietzel J, Thieme J, Eusterhues K, Eichert D (2007). Iron speciation in soils and soil aggregates by synchrotron-based X-ray microspectroscopy (XANES, $\mu$-XANES). European Journal of Soil Science, 58 (5): 1027-1041

Radke B, Jewell L, Piketh S, Namieśnik J (2014). Arsenic-based warfare agents: Production, use, and destruction. Critical Reviews in Environmental Science and Technology, 44(14): 1525-1576

Regelink I C, Voegelin A, Weng L, Koopmans G F, Comans R N (2014). Characterization of colloidal $\mathrm{Fe}$ from soils using field-flow fractionation and $\mathrm{Fe}$ K-edge X-ray absorption spectroscopy. Environmental Science \& Technology, 48(8): 4307-4316

Schwertmann U (1966). Inhibitory effect of soil organic matter on the crystallization of amorphous ferric hydroxide. Nature, 212(5062): 645-646

Sherman D M, Randall S R (2003). Surface complexation of arsenic(V) to iron(III) (hydr)oxides: Structural mechanism from ab initio molecular geometries and EXAFS spectroscopy. Geochimica et Cosmochimica Acta, 67(22): 4223-4230

Shimizu M, Arai Y, Sparks D L (2011). Multiscale assessment of methylarsenic reactivity in soil. 1. Sorption and desorption on soils. Environmental Science \& Technology, 45(10): 4293-4299

Tanaka M, Togo Y S, Yamaguchi N, Takahashi Y (2014). An EXAFS study on the adsorption structure of phenyl-substituted organoarsenic compounds on ferrihydrite. Journal of Colloid and Interface Science, 415: $13-17$

ThomasArrigo L K, Byrne J M, Kappler A, Kretzschmar R (2018). Impact of organic matter on iron(II)-catalyzed mineral transformations in ferrihydrite-organic matter coprecipitates. Environmental Science \& Technology, 52(21): 12316-12326

Wada K, Higashi T (1976). The categories of aluminium- and ironhumus complexes in Ando soils determined by selective dissolution. Journal of Soil Science, 27(3): 357-368

Wang A N, Li S X, Teng Y, Liu W X, Wu L H, Zhang H B, Huang Y J, Luo Y M, Christie P (2013). Adsorption and desorption characteristics of diphenylarsenicals in two contrasting soils. Journal of Environmental Sciences (China), 25(6): 1172-1179

Wang S, Mulligan C N (2008). Speciation and surface structure of inorganic arsenic in solid phases: A review. Environment Interna- 
tional, 34(6): 867-879

Weber F A, Hofacker A F, Voegelin A, Kretzschmar R (2010). Temperature dependence and coupling of iron and arsenic reduction and release during flooding of a contaminated soil. Environmental Science \& Technology, 44(1): 116-122

Zhu M, Hu X F, Tu C, Luo Y M, Yang R Y, Zhou S B, Cheng N N, Rylott E L (2019a). Speciation and sorption structure of diphenylarsinic acid in soil clay mineral fractions using sequential extraction and EXAFS spectroscopy. Journal of Soils and Sediments, 20(2): 763-764

Zhu M, Hu X F, Tu C, Zhang H B, Song F, Luo Y M, Christie P (2019b). Sorption mechanisms of diphenylarsinic acid on ferrihydrite, goethite and hematite using sequential extraction, FTIR measurement and XAFS spectroscopy. Science of the Total Environment, 669: 9911000

Zhu M, Tu C, Hu X F, Zhang H B, Zhang L J, Wei J, Li Y, Luo Y M, Christie P (2016a). Solid solution partitioning and thionation of diphenylarsinic acid in a flooded soil under the impact of sulfate and iron reduction. Science of the Total Environment, 569-570: 15791586

Zhu M, Tu C, Zhang H B, Luo Y M, Christie P (2016b). Simultaneous determination of diphenylarsinic and phenylarsinic acids in amended soils by optimized solvent extraction coupled to HPLC-MS/MS. Geoderma, 270: 109-116 